\title{
Study on the critical stress threshold of weakly cemented sandstone damage based on the renormalization group method
}

\author{
Zhaoyang Song ${ }^{1,2} \cdot$ Hongguang $\mathrm{Ji}^{2} \cdot$ Zhiqiang Liu $^{1} \cdot$ Lihui Sun $^{3}$
}

Received: 16 May 2019/Revised: 12 January 2020/Accepted: 27 March 2020/Published online: 15 April 2020

(C) The Author(s) 2020

\begin{abstract}
During the microstructural analysis of weakly cemented sandstone, the granule components and ductile structural parts of the sandstone are typically generalized. Considering the contact between granules in the microstructure of weakly cemented sandstone, three basic units can be determined: regular tetrahedra, regular hexahedra, and regular octahedra. Renormalization group models with granule- and pore-centered weakly cemented sandstone were established, and, according to the renormalization group transformation rule, the critical stress threshold of damage was calculated. The results show that the renormalization model using regular octahedra as the basic units has the highest critical stress threshold. The threshold obtained by iterative calculations of the granule-centered model is smaller than that obtained by the pore-centered model. The granule-centered calculation provides the lower limit (18.12\%), and the pore-centered model provides the upper limit $(36.36 \%)$. Within this range, the weakly cemented sandstone is in a phase-like critical state. That is, the state of granule aggregation transforms from continuous to discrete. In the relative stress range of $18.12 \%-36.36 \%$, the weakly cemented sandstone exhibits an increased proportion of high-frequency signals (by 83.3\%) and a decreased proportion of low-frequency signals (by 23.6\%). The renormalization calculation results for weakly cemented sandstone explain the high-low frequency conversion of acoustic emission signals during loading. The research reported in this paper has important significance for elucidating the damage mechanism of weakly cemented sandstone.
\end{abstract}

Keywords Weakly cemented sandstone $\cdot$ Renormalization group method - Damage and failure $\cdot$ Threshold

\section{Introduction}

The Ordos and Yulin areas of western China exhibit typical lithologies for the region, with the strata mainly composed of weakly cemented Jurassic sandstone. On the microscopic scale, weakly cemented sandstone consists of three components: granules, cementation, and pores. The

\section{Zhaoyang Song}

szhaoyang123@126.com

1 Beijing China Coal Mine Engineering Company Limited, Beijing 100013, China

2 School of Civil and Resource Engineering, University of Science and Technology Beijing, Beijing 100083, China

3 School of Mining and Geomatics, Hebei University of Engineering, Handan 056038, China proportion of particulate matter in weakly cemented sandstone is $85.75 \%$, and the proportion of cementitious material is $9.4 \%$. The granules are mainly aggregated by compaction and cementation, and the granule skeleton of cemented material with similar functions can be described from its macrostructure to its microstructure ( $\mathrm{Ji}$ et al. 2018). By analyzing the mineral composition of weakly cemented sandstone, it appears that the cemented material is mainly composed of argillaceous cement, has low strength, and is not resistant to pressure or tension ( Liu and Qin 2019). There is no single characteristic scale for weakly cemented sandstone, whether it fails via the destruction of the cemented minerals or theformation of macroscopic fractures. Therefore, weakly cemented sandstone can be classified as a particulate material (Song et al. 2018a, b). When analyzed from the macroscale, there must be a certain stability threshold that can describe the 
behavior of a group of cemented granules. After weakly cemented sandstone has been subdivided at several scales, each part can be scaled and compared with the original, and the two will appear similar. That is, the part and the whole are similar in form, function, and information. Therefore, weakly cemented sandstone has the property of self-similarity, which is the most important physical feature considered in the renormalization group method (Ji 2004). During the loading process of weakly cemented coarsegrained sandstone, the microcracks of the microstructure expand as a fractal structure (Liu et al. 2019). In the critical damage state, the crack penetration of cemented sandstone is scale invariant. That is, the critical damage has the same destructive properties at different scales. Therefore, the renormalization group method is suitable for the analysis of weakly cemented sandstone materials.

To a certain extent, the complexity of geotechnical material problems is due to the structural properties of the geomaterials. Physically, the macroscopic mechanical scale and microstructural scale of weakly cemented sandstone are interdependent. However, the corresponding physical properties change with the scale. The relations among the physical properties of weakly cemented sandstone at different scales are a key topic of study. The renormalization group method has an important role in this research. The concept of the renormalization group originated from quantum field theory. To address the divergence problem in quantum field theory, a renormalization method was developed to avoid singularities (Weinberg 1995). Wilson (1971a, b) used the renormalization group method to study continuous phase transitions, and found that the self-similarity exhibited by the system at different scales near the critical point could be described by the renormalization group method (Goldenfeld 1992). This discovery provides a more reasonable and effective theoretical expression for continuous phase transition, as well as a more direct physical basis for the renormalization group method. Previous studies have shown that particulate materials exhibit extremely complex mechanical behavior during loading and important properties appear in the critical states (Casagrande 1936). The concept of critical states was established a long time ago. Although most scholars still describe critical states from a macroscopic perspective, some important results have been obtained from the perspective of the microstructure. Rothenburg and Kruyt (2004) used the coordination number (the number of contacts around each granule) to characterize the microscopic critical state of particulate material, and studied the effect of the granule surface friction coefficient on the critical coordination number (the coordination number at the critical state). Zhang and Qin (2008) and Qin and Zhang (2010) used the contact valence bond (the number of sides of each pore cell) to characterize the microscopic critical state, and noted that the parameters characterizing this state depend on the microscopic properties of the granules, such as the granule shape, surface friction properties, initial pore ratio, and granule confining pressure. Renormalization groups can be used to address problems involving multiple length scales, whereby the renormalization group method processes the problem step by step, with each length scale representing a single step. The technical problem is the statistical averaging of thermal fluctuations for all scales. The renormalization group method has been applied to phase transitions, and scholars have established the renormalization group method for this critical phenomenon (Gao et al. 2009). Using the renormalization group method, the relationship between the selforganized characteristics of the rock failure process and the brittle rock deformation mechanism and permeability can be studied. The critical criteria for elucidating the microstructural evolution of soft rock are descriptions of the water softening process and the fractal and percolation evolution characteristics during rock damage, which provide the essential correlation between the percolation and damage during soft rock damage (Zhou et al. 2015; Li et al. 2019).

In summary, the renormalization group method is an effective means of studying the critical eigenvalues of geomaterials. In this paper, by analyzing the microstructure of weakly cemented sandstone, the granular and cemented components are determined; the three basic unit forms of regular tetrahedra, regular hexahedra, and regular octahedra are established; and a renormalization group model of weakly cemented sandstone is developed. The critical stress threshold for weakly cemented sandstone damage is studied, and the results effectively explain the conversion between high- and low-frequency signals of acoustic emissions during the weakly cemented sandstone loading process.

\section{Establishing a renormalization group model for weakly cemented sandstone}

\subsection{Renormalization transformation method}

When a research material exhibits good similarity, the mode of the renormalization transformation is relatively fixed. Thus, the transformation manner is the same across all scales, making the transformation relatively simple (Zhou et al. 2015).

Taking a material system as the research object, observations are carried out at scale $l_{1}$ to obtain a certain physical quantity $T_{1}$ of the system; when the scale is increased to $l_{2}$, the corresponding physical quantity of the 
system changes from $T_{1}$ to $T_{2}$ via a nonlinear transformation. This change can be expressed as follows:

$T_{2}=f\left(T_{1}\right)$

As the scale increases,

$T_{n+1}=f\left(T_{n}\right)(n=1,2,3 \ldots)$

According to Wilson's assumption (Wilson 1971a, b), $f_{i} \cdot f_{i}=f_{i+j}$, and $f_{i}=I$, where $i, j=1,2,3 \ldots$.

Then

$T_{n+1}=f_{n}\left(T_{1}\right)(n=1,2,3 \ldots)$

When $n$ is sufficiently large, the following can be obtained according to renormalization theory:

$T_{\mathrm{c}}=f_{n}\left(T_{\mathrm{c}}\right)$

That is, $T_{\mathrm{c}}$ is a nonlinear fixed point.

Iterating around the fixed point, there are two main cases: (1) the calculated value is farther from the fixed point after iteration; (2) after iteration, the calculated value is closer to the fixed point. The former reflects an unstable fixed point, whereas the latter reflects a stable fixed point. The critical point of a critical phenomenon is the unstable fixed point (He et al. 2010).

\subsection{Establishment of renormalization group model for weakly cemented sandstone}

The microstructure of weakly cemented sandstone was obtained by scanning electron microscopy, and the test results are shown in Fig. 1. In Fig. 1, the granules of the weakly cemented sandstone have different contact modes (such as face contact, occlusal contact, point contact, pore contact, and cementation contact), and the cementation is mainly clay minerals. Previous studies have shown that the strength of weakly cemented sandstone depends on the strength of the cemented material (Song and Ning 2018). A stronger cementitious material in the rock results in higher rock strength, and vice versa. The microstructure of the weakly cemented sandstone acts as a skeleton, and the clay mineral content acts as cement (Song et al. 2018a, b).
Moreover, similar granule-forming skeletons and cementing materials can be found in both the microstructure and macrostructure of weakly cemented sandstone. Therefore, the basic unit of the renormalized group model of weakly cemented sandstone can be established.

In the process of scale change, it is assumed that the substances acting as the skeleton and cement can always be identified, and that only the basic unit of the upper scale undergoes cement failure. During the loading process of weakly cemented sandstone, the granular units are rigid, and the cemented units undergo compressive, tensile, and shear failure. This causes the structural elements to be displaced, resulting in structural changes of the weakly cemented sandstone. The structural changes of weakly cemented sandstone cause the fracture of the rock sample. However, fracturing of the rock sample can be classified as a stability problem or a phase transition problem in the material of the rock skeleton (Song 2017; Wei 2012).

Based on the above analysis of the contact mode and type of granules within the microstructure of weakly cemented sandstone, the microstructural components were determined to have the form shown in Fig. 2. In this figure, the composite component of the weakly cemented sandstone microstructure comprises two rigid granule units and one cementing unit.

In the three-dimensional infinite space, only regular tetrahedra, regular hexahedra, and regular octahedra can construct a variety of solids and exhibit translational symmetry, as dodecahedra and icosahedra cannot be stacked into crystals. In this paper, based on the component unit and different combination modes, the basic units of the granule- and pore-centered renormalization models are constructed.

The basic units of the granule-centered renormalization model for weakly cemented sandstone are shown in Fig. 3. As shown in Fig. 3a, the basic structural unit in the form of a regular tetrahedron is composed of one granular member and nine clay structural members. As shown in Fig. 3b, the basic structural unit of the regular hexahedron consists of one granular member and six clay structural members. As
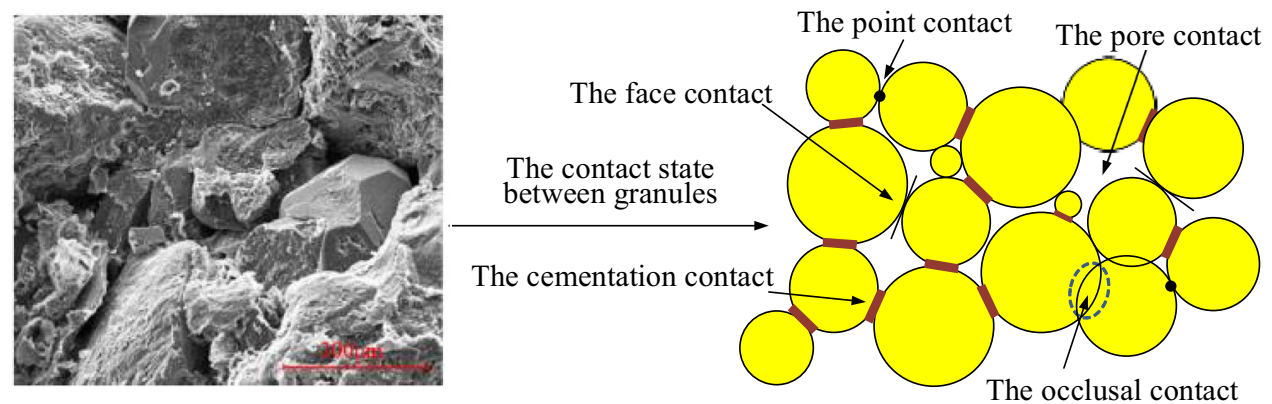

Fig. 1 Microscopic structure of weakly cemented sandstone 
shown in Fig. 3c, the basic structural unit of the regular octahedron is composed of one granular member and eight clay structural members.

The basic units of the pore-centered renormalization model for weakly cemented sandstone are shown in Fig. 4. As shown in Fig. 4a, the basic structural unit of the regular tetrahedron is composed of four granule members and six clay members centered on the pores. As shown in Fig. 4b, the basic structural unit of the regular hexahedron is composed of eight granular members and 12 clay members centered on the pores. As shown in Fig. 4c, the basic structural unit of the regular octahedron is composed of six granular members and 12 clay members centered on the pores.

The rigid granule unit The rigid granule unit

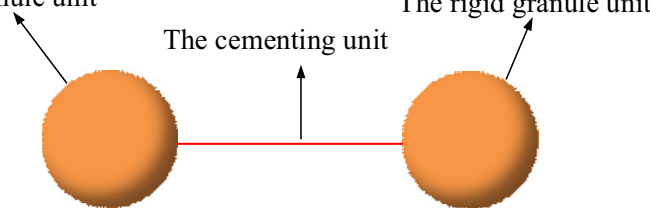

Fig. 2 Basic components of the microstructure of weakly cemented sandstone
The basic units are combined at different scales to establish a renormalized group model of weakly cemented sandstone, as shown in Fig. 5.

\subsection{Renormalization model variation rules for weakly cemented sandstone under load conditions}

To simplify the calculation, the renormalization model makes the following assumptions: (1) the renormalization model is isotropic; (2) the failure probabilities of the cement components are equal at the same scale.

The weakly cemented sandstone is characterized by internal micro-cracks that extend through the fractal structure, causing instability. As shown in Figs. 3 and 4, the ductile structural parts of the microstructural units of the weakly cemented sandstone are destroyed, and the symmetrical structure is seriously altered, which directly leads to structural instability. That is, the crack penetrates along the surface of the granules, and the granules are prone to spall. The specific rules of the weakly cemented sandstone renormalization transformation are shown in Figs. 6 and 7. The granule-centered microstructural unit transformation rules for weakly cemented sandstone are shown in Fig. 6,

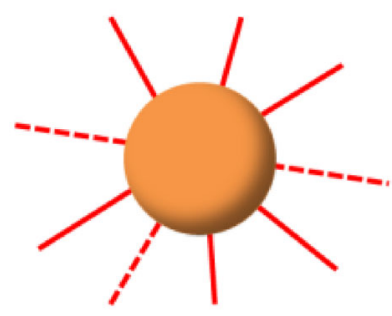

(a) Regular tetrahedron

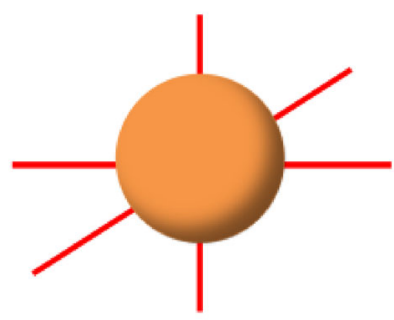

(b) Regular hexahedron

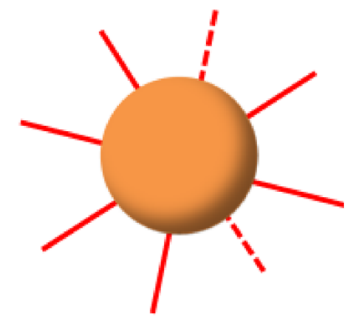

(c) Regular octahedron

Fig. 3 Granule-centered microstructural units of the weakly cemented sandstone: a regular tetrahedron, b regular hexahedron, c regular octahedron
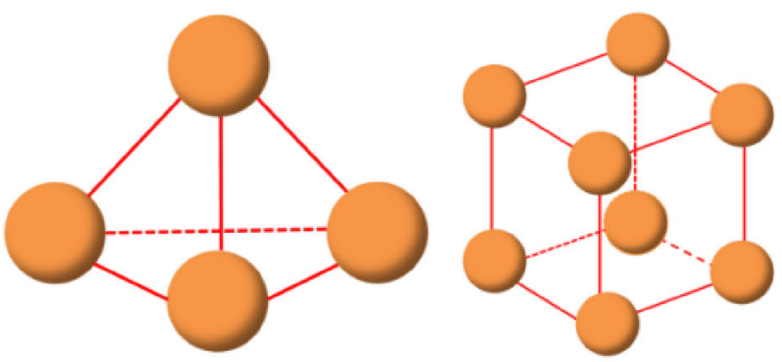

(b) Regular hexahedron (a) Regular tetrahedron

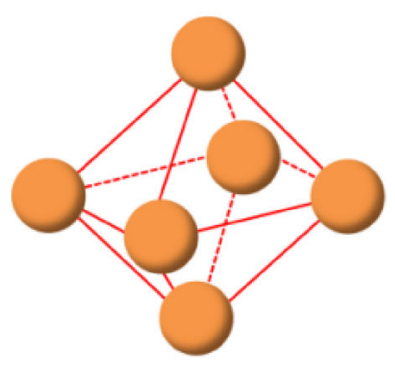

(c) Regular octahedron

Fig. 4 Pore-centered microstructural units of the weakly cemented sandstone: a regular tetrahedron, b regular hexahedron, c regular octahedron 


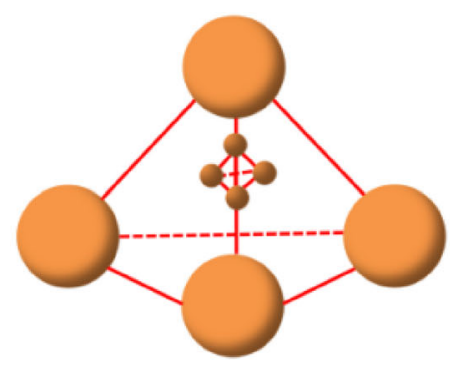

(a) Regular tetrahedron

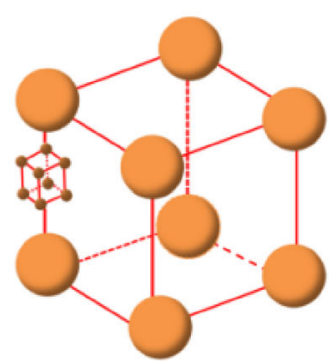

(b) Regular hexahedron

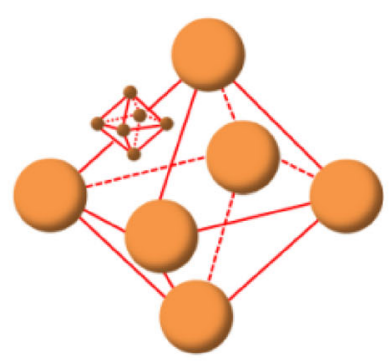

(c) Regular octahedron

Fig. 5 Renormalization group models of the weakly cemented sandstone: a regular tetrahedron, $\mathbf{b}$ regular hexahedron, $\mathbf{c}$ regular octahedron
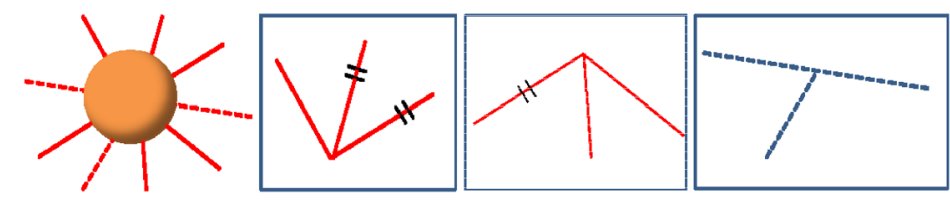

Transformation rule: When at least 3 cementing units are broken, it is considered that the crack penetrates along the particles and appears as damage of the cement member at the next level.

(a) Regular tetrahedron

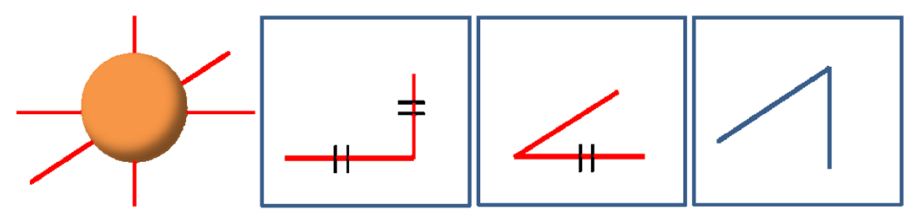

(b) Regular hexahedron

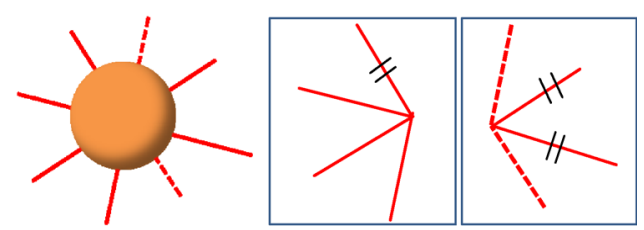

Transformation rule: In each group of 4 cementing units, when at least 3 units are broken, the damage of the cementing units is considered, and the damage of the cement member is exhibited at the next level.

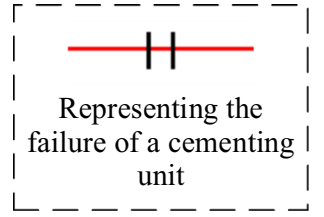

(c) Regular octahedron

Fig. 6 Destruction rule of granule-centered microstructural units of the weakly cemented sandstone: a regular tetrahedron, $\mathbf{b}$ regular hexahedron, c regular octahedron

and the transformation rule of the microstructural units centered on pores is shown in Fig. 7.

\section{Calculation of the damage critical stress value of weakly cemented sandstone}

\subsection{Damage threshold of the granule-centered model}

According to the renormalization group model and transformation rules of weakly cemented sandstone, the damage types of the basic elements in the renormalization group model are calculated. The types of failure of the weakly cemented sandstone with granule-centered structural units are presented in Table 1.

Taking a regular hexahedral structural unit as an example (as shown in Fig. 6b), the basic unit is composed of six cement members and one skeleton member, wherein the cement member may exhibit 0-6 kinds of damage. According to the rules and probability calculations of the renormalization changes, when the ductile structural members are destroyed by $0-2$ kinds of damage, the unit has no failure types. When the ductile structural member is destroyed by three kinds of damage, the number of failure types of the unit is 


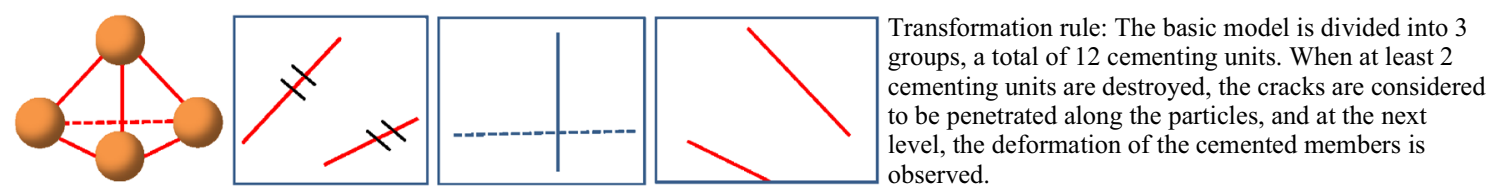

(a) Regular tetrahedron
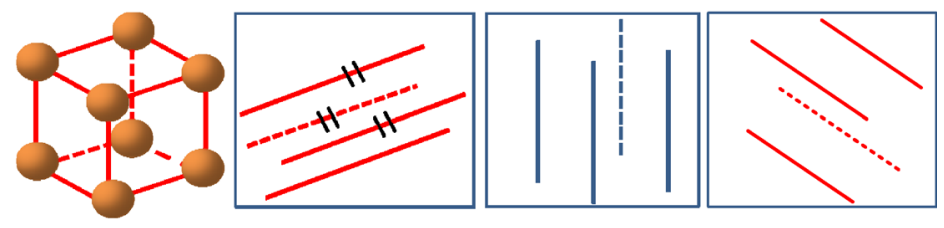

Transformation rule: The basic model is divided into 3 groups, a total of 12 cementing units. When at least 3 cementing units are destroyed, the cracks are considered to be penetrated along the particles, and at the next level, the deformation of the cemented members is observed.

(b) Regular hexahedron
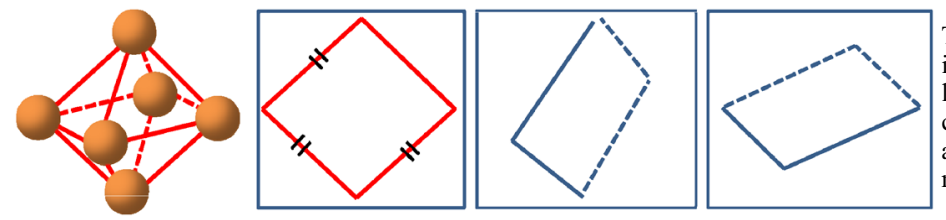

Transformation rule: The basic model is divided into 3 groups, a total of 12 cementing units. When at least 3 cementing units are destroyed, the cracks are considered to be penetrated along the particles, and at the next level, the deformation of the cemented members is observed.

(c) Regular octahedron

Fig. 7 Pore-centered microstructural units of weakly cemented sandstone: a regular tetrahedron, $\mathbf{b}$ regular hexahedron, $\mathbf{c}$ regular octahedron

Table 1 Failure types of granule-centered microstructural units in weakly cemented sandstone

\begin{tabular}{|c|c|c|c|c|c|}
\hline \multicolumn{2}{|l|}{ Regular tetrahedra } & \multicolumn{2}{|l|}{ Regular hexahedra } & \multicolumn{2}{|l|}{ Regular octahedra } \\
\hline $\begin{array}{l}\text { Number of pieces of } \\
\text { ductile structural units that } \\
\text { undergo damage }\end{array}$ & $\begin{array}{l}\text { Number of } \\
\text { types of damage } \\
\text { to the unit }\end{array}$ & $\begin{array}{l}\text { Number of pieces of } \\
\text { ductile structural units that } \\
\text { undergo damage }\end{array}$ & $\begin{array}{l}\text { Number of } \\
\text { types of damage } \\
\text { to the unit }\end{array}$ & $\begin{array}{l}\text { Number of pieces of } \\
\text { ductile structural units that } \\
\text { undergo damage }\end{array}$ & $\begin{array}{l}\text { Number of } \\
\text { types of damage } \\
\text { to the unit }\end{array}$ \\
\hline 0 & 0 & 0 & 0 & 0 & 0 \\
\hline 1 & 0 & 1 & 0 & 1 & 0 \\
\hline 2 & 0 & 2 & 0 & 2 & 0 \\
\hline 3 & 219 & 3 & 60 & 3 & 56 \\
\hline 4 & 234 & 4 & 27 & 4 & 106 \\
\hline 5 & 234 & 5 & 6 & 5 & 56 \\
\hline 6 & 222 & 6 & 1 & 6 & 44 \\
\hline 7 & 72 & - & - & 7 & 8 \\
\hline 8 & 18 & - & - & 8 & 1 \\
\hline 9 & 1 & - & - & - & - \\
\hline Total & 1000 & Total & 94 & Total & 271 \\
\hline
\end{tabular}

$$
\mathrm{C}_{3}^{1} \mathrm{C}_{2}^{2} \mathrm{C}_{2}^{1} \mathrm{C}_{2}^{1}+\mathrm{C}_{3}^{1} \mathrm{C}_{2}^{1} \mathrm{C}_{2}^{1} \mathrm{C}_{2}^{1} \mathrm{C}_{2}^{1}=60
$$

When there are four kinds of damage to the structural ductile parts, the number of failures of the unit is

$\mathrm{C}_{3}^{1} \mathrm{C}_{2}^{2}+\mathrm{C}_{3}^{1} \mathrm{C}_{2}^{1} \mathrm{C}_{2}^{1} \mathrm{C}_{2}^{1}=27$

When there are five kinds of damage to the ductile structural member, the number of failure types of the unit is
$\mathrm{C}_{3}^{1} \mathrm{C}_{2}^{1}=6$

When the ductile structural member is broken by six kinds of damage, there is one type of failure of the unit. A total of 94 failure types causing unit destruction are obtained. Using the mathematical combination method, the types of damage of regular tetrahedral structural units and 
regular octahedral structural units can also obtained in Table 1.

It is assumed that the probability of failure of the initial cement member is $Q$ and that the probability that the cement member is not destroyed is $1-Q$. The probability of unit destruction at the first-order scale of the hexahedral structure is

$$
\begin{aligned}
Q_{1}= & 60 Q^{3} \times(1-Q)^{3}+27 Q^{4} \times(1-Q)^{2}+6 Q^{5} \\
& \times(1-Q)^{1}+Q^{6}
\end{aligned}
$$

Equation (8) can be rearranged to obtain

$Q_{1}=-38 Q^{6}+132 Q^{5}-153 Q^{4}+60 Q^{3}$

According to renormalization group theory, the unit destruction probability can be written at scale $l_{n}$ as

$Q_{n}=-38 Q_{n-1}^{6}+132 Q_{n-1}^{5}-153 Q_{n-1}^{4}+60 Q_{n-1}^{3}$

When $n$ is sufficiently large, there is a fixed point $Q_{n}$ such that

$Q_{n}=Q_{n-1}$

Equations (10) and (11) were combined and solved in MATLAB. The calculation results are shown in Fig. 8. According to the results, the fixed points are 0, 0.1609, and 1. In the same way, the fixed points of the granule-centered regular tetrahedron and regular octahedron models were determined, and the results are shown in Fig. 9.

When the absolute value of the slope of the fixed point is less than unity, the iteratively calculated value will be closer to the fixed point, indicating a stable fixed point. When the absolute value of the slope of the fixed point is greater than unity, the iteratively calculated value will increasingly deviate from the fixed point, indicating an unstable fixed point. Unstable motion is the critical point of the damage state. Therefore, from the above solution

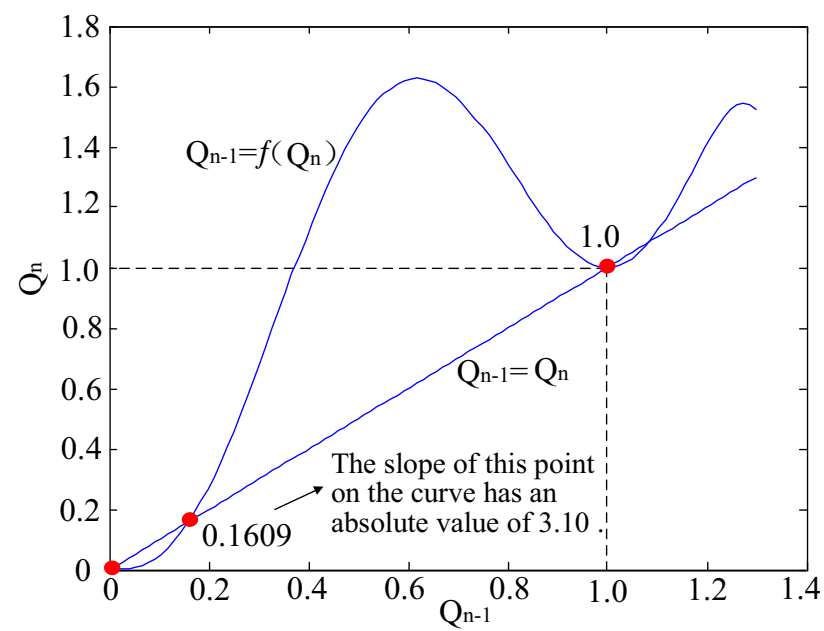

Fig. 8 Solution of the fixed points for the granule-centered regular hexahedron

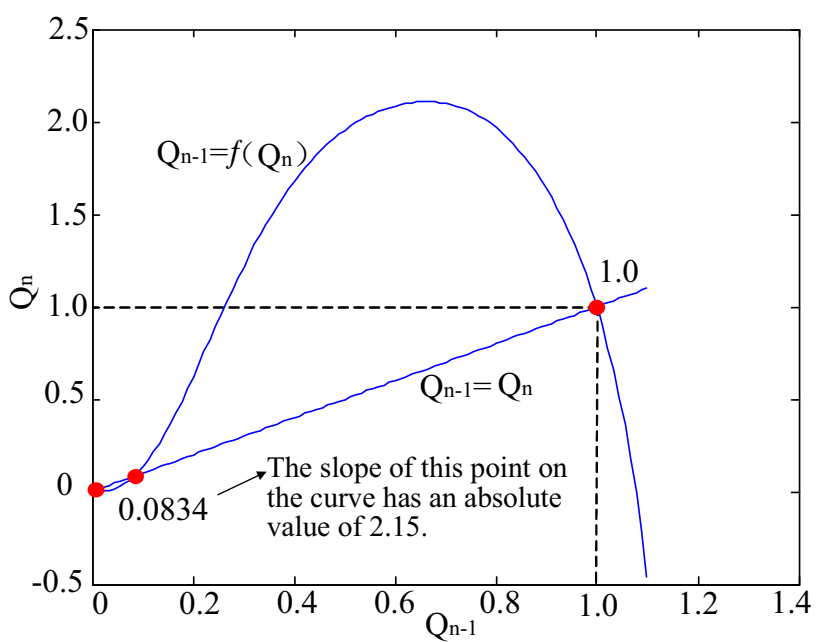

(a) Regular tetrahedron

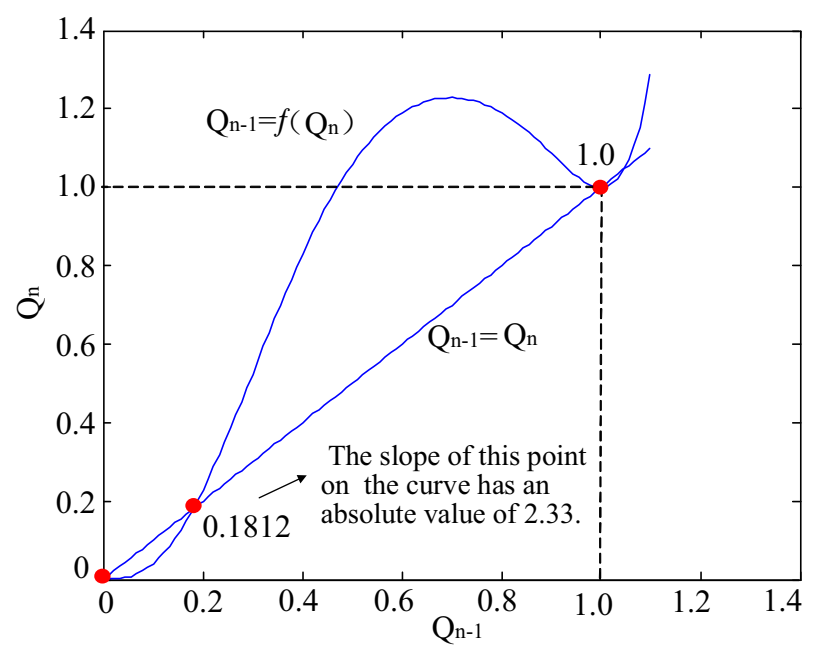

(b) Regular octahedron

Fig. 9 Solution of the fixed points in the granule-centered regular hexahedron and regular octahedron

Table 2 Absolute values of the slopes of fixed points in different granule-centered structures

\begin{tabular}{lll}
\hline Structural units & Fixed point & Absolute value of the fixed point \\
\hline Regular tetrahedron & 0.0834 & 2.15 \\
$\begin{array}{l}\text { Regular } \\
\quad \text { hexahedron }\end{array}$ & 0.1609 & 3.10 \\
Regular octahedron & 0.1812 & 2.33 \\
\hline
\end{tabular}

process and Figs. 8 and 9, the fixed points of the granulecentered renormalization model of the weakly cemented sandstone were obtained, and the results are presented in Table 2. 
Table 3 Failure types of pore-centered microstructural units in weakly cemented sandstone

\begin{tabular}{|c|c|c|c|c|c|}
\hline \multicolumn{2}{|l|}{ Regular tetrahedron } & \multicolumn{2}{|l|}{ Regular hexahedron } & \multicolumn{2}{|l|}{ Regular octahedron } \\
\hline $\begin{array}{l}\text { Number of pieces of } \\
\text { ductile structural } \\
\text { damage }\end{array}$ & $\begin{array}{l}\text { Number of types of } \\
\text { damage to the unit }\end{array}$ & $\begin{array}{l}\text { Number of pieces of } \\
\text { ductile structural } \\
\text { damage }\end{array}$ & $\begin{array}{l}\text { Number of types of } \\
\text { damage to the unit }\end{array}$ & $\begin{array}{l}\text { Number of pieces of } \\
\text { ductile structural } \\
\text { damage }\end{array}$ & $\begin{array}{l}\text { Number of types of } \\
\text { damage to the unit }\end{array}$ \\
\hline 0 & 0 & 0 & 0 & 0 & 0 \\
\hline 1 & 0 & 1 & 0 & 1 & 0 \\
\hline 2 & 27 & 2 & 0 & 2 & 0 \\
\hline 3 & 60 & 3 & 12 & 3 & 12 \\
\hline 4 & 30 & 4 & 99 & 4 & 99 \\
\hline 5 & 12 & 5 & 360 & 5 & 360 \\
\hline 6 & 1 & 6 & 708 & 6 & 708 \\
\hline- & - & 7 & 792 & 7 & 792 \\
\hline- & - & 8 & 495 & 8 & 495 \\
\hline- & - & 9 & 220 & 9 & 220 \\
\hline- & - & 10 & 66 & 10 & 66 \\
\hline- & - & 11 & 12 & 11 & 12 \\
\hline- & - & 12 & 1 & 12 & 1 \\
\hline Total & 130 & Total & 2765 & Total & 2765 \\
\hline
\end{tabular}

\subsection{Damage threshold of the pore-centered model}

According to the renormalization group model and transformation rules of weakly cemented sandstone, the damage types of the basic elements of the renormalization group model were calculated. The failure types of weakly cemented sandstone with pore-centered structural units are listed in Table 3.

Taking a regular hexahedral structural unit as an example (as shown in Fig. 7b), the basic unit is composed of 12 cement members and eight skeleton members, wherein the cement member exhibits $0-12$ kinds of damage. According to the rules and probability calculations of the renormalization changes, the calculation method is the same as for the body element failure type of the positive six-sided surface in Table 1, and the mathematical combination algorithm is adopted. A total of 2765 failure types that cause unit destruction are obtained.

It is assumed that the probability of failure of the initial cement member is $P$ and that the probability that the cement member is not destroyed is 1-P. The probability of unit destruction at the first-order scale of the hexahedral structure is

$$
\begin{aligned}
P_{1}= & 12 P^{3}(1-P)^{9}+99 P^{4}(1-P)^{8}+360 P^{5}(1-P)^{7} \\
& +216 P^{6}(1-P)^{6}+792 P^{7}(1-P)^{5}+495 P^{8}(1-P)^{4} \\
& +220 P^{9}(1-P)^{3}+66 P^{10}(1-P)^{2}+12 P^{11}(1-P) \\
& +P^{12}
\end{aligned}
$$

Equation (12) can be rearranged to obtain

$$
\begin{aligned}
P_{1}= & -27 P^{12}+108 P^{11}-144 P^{10}+64 P^{9}-27 P^{8}+72 P^{7} \\
& -48 P^{6}-9 P^{4}+12 P^{3}
\end{aligned}
$$

According to the renormalization group theory, the unit destruction probability can be written at scale $l_{n}$ as

$$
\begin{aligned}
P_{n}= & -27 P_{n-1}^{12}+108 P_{n-1}^{11}-144 P_{n-1}^{10}+64 P_{n-1}^{9}-27 P_{n-1}^{8} \\
& +72 P_{n-1}^{7}-48 P_{n-1}^{6}-9 P_{n-1}^{4}+12 P_{n-1}^{3}
\end{aligned}
$$

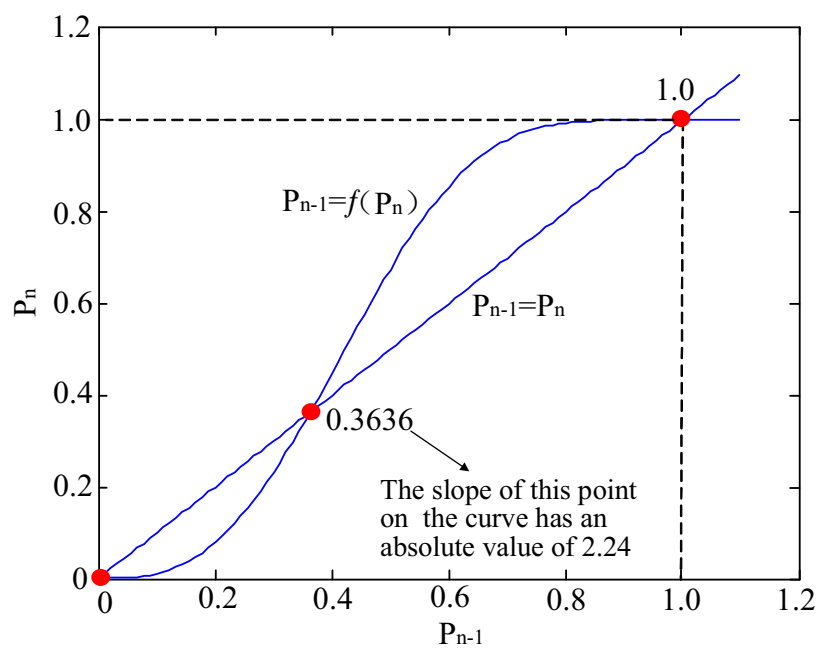

Fig. 10 Solutions to the fixed points of the pore-centered regular hexahedron 


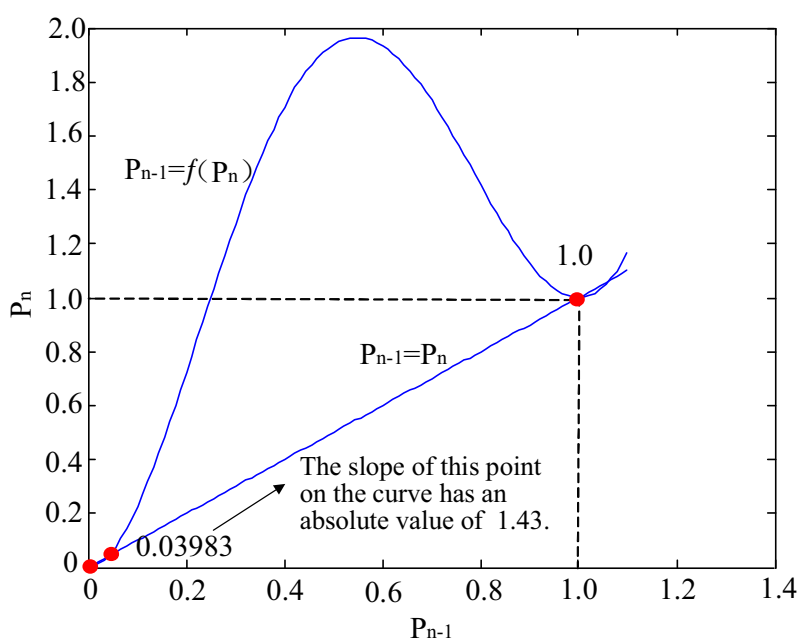

(a) Regular tetrahedron

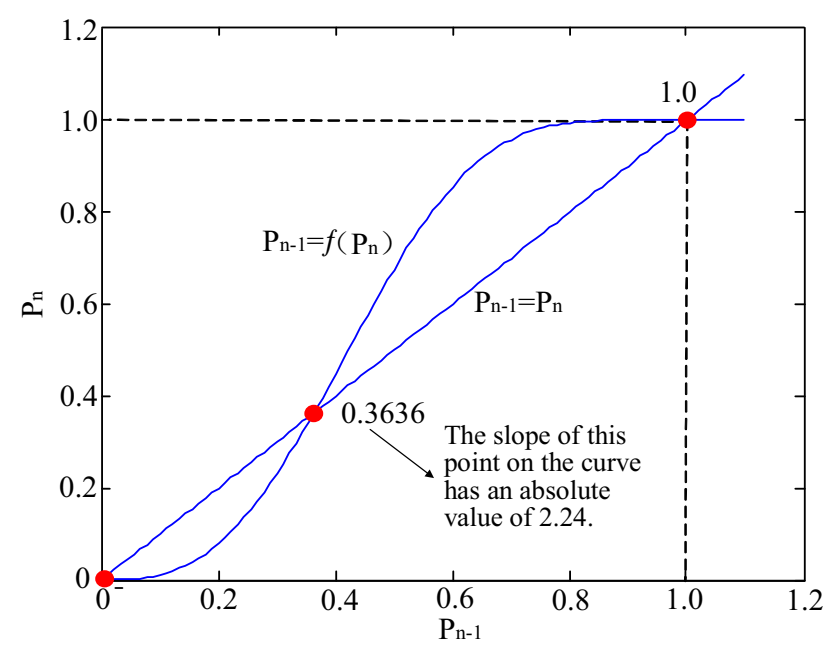

(b) Regular octahedron

Fig. 11 Solution of the fixed points of the pore-centered regular hexahedron and regular octahedron

Table 4 Calculated solutions to the fixed points in the pore-centered microstructural units of weakly cemented sandstone

\begin{tabular}{lll}
\hline Structural units & Fixed point & Absolute value of the fixed point \\
\hline Regular tetrahedron & 0.03983 & 1.43 \\
$\begin{array}{l}\text { Regular } \\
\text { hexahedron }\end{array}$ & 0.36360 & 2.24 \\
$\begin{array}{l}\text { Regular octahedron } \\
\text { N }\end{array}$ & 0.36360 & 2.24 \\
\hline
\end{tabular}

When $n$ is sufficiently large, there is a fixed point $Q_{n}$ such that

$P_{n}=P_{n-1}$

Equations (14) and (15) were combined and solved in MATLAB. The calculation results are shown in Fig. 10.
According to the results, the fixed points are $0,0.3636$, and 1. In the same way, the fixed points of the pore-centered regular tetrahedron and regular octahedron models were determined, and the results are shown in Fig. 11.

When the absolute value of the slope of the fixed point is less than unity, the iterative calculated value will be closer to the fixed point, indicating a stable fixed point. When the absolute value of the slope of the fixed point is greater than unity, the iteratively calculated value will increasingly deviate from the fixed point, indicating an unstable fixed point. Unstable motion is the critical point of the damage state. Therefore, from the above solution and Figs. 10 and 11 , the fixed points of the pore-centered renormalization model of weakly cemented sandstone were obtained, and the results are presented in Table 4.

\section{Discussion}

With the pore-centered and granule-centered renormalization models, the critical value of the damage of weakly cemented sandstone during the loading process was calculated. However, the combination of granules in weakly cemented sandstone is a collection of multiple structural forms. In this study, different critical stress thresholds were obtained with regular tetrahedral, hexahedral and octahedral structural models. Therefore, a rational and scientific definition of the critical stress threshold of weakly cemented sandstone is possible.

According to the physical meaning, assumptions, and failure rules of the weakly cemented sandstone renormalization group models, destruction only occurs in the cemented matter between the granules. When the weakly cemented sandstone reaches the point of critical damage, the granules are still in continuous contact, and static friction only occurs between the granules. At this time, the skeleton structure, which is composed of granules, still has a certain bearing capacity. When the weakly cemented sandstone is loaded, damage accumulates continuously, and the destruction of the cemented material changes the granule state in the microstructure of the weakly cemented sandstone, thus changing the microstructure system. Therefore, during the loading process, the granule aggregation state is considered to transform from a continuous state to a discrete state. This process is called the critical phase transition of weakly cemented sandstone.

According to the calculation results, the upper limit given by the pore-centered model and the lower limit given by the granule-centered model are selected to provide the interval of the critical phase transition state for weakly cemented sandstone. This interval is 0.1812-0.3636. According to this definition, when the degree of damage of weakly cemented sandstone is less than $18.12 \%$, the state 
of granule aggregation is continuous; when the degree of damage is greater than $36.36 \%$, the local granules in the shear zone have become discrete. In this state, due to the action of the external pressure, the granules inside the weakly cemented sandstone remain in contact, and the sample is in a metastable state. Although the sample has a certain load-carrying capacity at this time, it is extremely sensitive to vibration or disturbance, and a slight strain $(\Delta \varepsilon)$ is likely to cause macroscopic cracking.

A previous study identified the characteristics of the frequency band of the acoustic emission signal during the damage of weakly cemented sandstone as a function of stress (Zeng and Li 2017). High-low frequency conversion occurs in the acoustic emission signal during the loading of weakly cemented sandstone. Among the signals, the proportion of low-frequency signals in the acoustic emission decreases, while the proportion of high-frequency signals increases. The specific results are presented in Fig. 12. As shown in the figure, in the relative stress interval from $18.12 \%$ to $36.36 \%$, the proportion of high-frequency acoustic emission signals increased by $83.3 \%$, and the proportion of low-frequency signals decreased by $23.6 \%$. The experimental results are in good agreement with the calculation results obtained by the renormalization group method. Therefore, the calculation results of the renormalization group method of weakly cemented sandstone effectively explain the high-low frequency conversion of acoustic emissions during the loading process.

As shown in Fig. 12, in the first phase, the relative stress is less than $18.12 \%$, and the pores or defects in the microstructure of the weakly cemented sandstone change slightly; the emitted acoustic emission signals are mainly low-frequency signals. In the second phase, the relative stress ranges from $18.12 \%$ to $36.36 \%$. In this stage, the cemented material in the microstructure of the weakly cemented sandstone is gradually destroyed, and the contact state of the granules changes from the cemented state to the

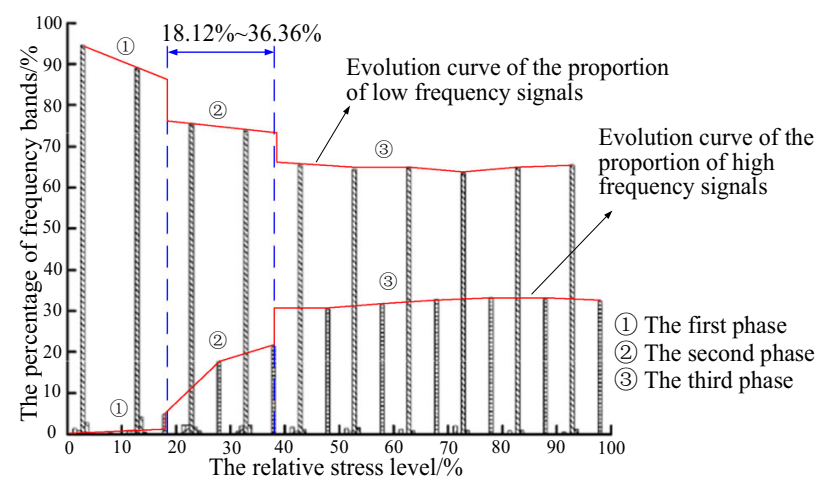

Fig. 12 Diagram of the low-high frequency conversion mode of acoustic emission signals before the peak stress of weakly cemented sandstone discrete state. In the third phase, the relative stress is greater than $36.36 \%$. The contact between the granules in the microstructure of the weakly cemented sandstone is rapidly reduced, the intrinsic failure mode of the sample changes, and slip and displacement occur between the granules, resulting in the generation of macroscopic cracks. At the same time, the proportion of high-frequency signals released reaches $31.9 \%$, with the proportion of low-frequency signals remaining at $66.8 \%$. In this phase, the damage rate of the rock sample intensifies, and its structure changes from stable damage to unsteady damage until it is completely destabilized.

\section{Conclusions}

By analyzing the microstructure of weakly cemented sandstone, the granular and cemented members of the weakly cemented sandstone have been generalized, and three basic unit forms of regular tetrahedra, regular hexahedra, and regular octahedra were established. Considering granule-centered and pore-centered structures, renormalization group models of weakly cemented sandstone were established, and the rules of renormalization transformation were derived.

The combination of granules in weakly cemented sandstone is a collection of multiple structural forms. Using the renormalization group method, different critical stress thresholds were obtained by the regular tetrahedral, hexahedral, and octahedral structure models. The critical stress threshold of weakly cemented sandstone damage obtained after iterative calculations of the basic octahedral element model was found to be higher than those of the other element models. The critical stress threshold obtained by iterative calculations of the granule-centered model is smaller than that obtained by the pore-centered model.

During the loading process of weakly cemented sandstone, the state of granule aggregation transforms from a continuous state to a discrete state, representing the critical phase transition. When the damage is less than $18.12 \%$, the aggregation state of the weakly cemented coarse sandstone granules is nearly continuous; when the damage degree is greater than $36.36 \%$, the local granules in the shear zone are discrete. At this time, the weakly cemented sandstone is in a metastable state and is sensitive to disturbances.

During the loading process of weakly cemented sandstone, the relative stress is in the range $18.12 \%-36.36 \%$, and the high-frequency signal ratio increases while the low-frequency signal ratio decreases. The proportion of high-frequency acoustic emission signals increases by $83.3 \%$, and the proportion of low-frequency signals decreases by $23.6 \%$. Therefore, the calculation results of the renormalization group method of weakly cemented 
sandstone provide a better explanation than other approaches of the high-low frequency conversion of acoustic emissions during the loading process.

Open Access This article is licensed under a Creative Commons Attribution 4.0 International License, which permits use, sharing, adaptation, distribution and reproduction in any medium or format, as long as you give appropriate credit to the original author(s) and the source, provide a link to the Creative Commons licence, and indicate if changes were made. The images or other third party material in this article are included in the article's Creative Commons licence, unless indicated otherwise in a credit line to the material. If material is not included in the article's Creative Commons licence and your intended use is not permitted by statutory regulation or exceeds the permitted use, you will need to obtain permission directly from the copyright holder. To view a copy of this licence, visit http://creativecommons. org/licenses/by/4.0/.

Acknowledgements This research was supported by the National Natural Science Foundation of China (Grant No. 51534002) and the Special Funds for Technological Innovation and Entrepreneurship of China Coal Science and Engineering Group Co. Ltd. (2018-TDMS011).

\section{References}

Casagrande A (1936) Characteristics of cohesion less soils affecting the stability of slopes and earth fills. J Boston Soc Civ Eng 23:257-276

Gao ZN, Yao LK, Xu GX (2009) Research on self-organization characteristics and critical conditions of rock failure process. J Sichuan Univ (Engineering Science Edition) 41(2):91-95

Goldenfeld N (1992) Lectures on phase transitions and the renormalization group. Addison-Wesley Publishing Company, Reading, pp 16-19

He C, Lv XL, Gao FY et al (2010) Re-normalization group method for calculation of seepage parameters of two-dimensional conductive points. J Appl Sci 28(2):170-174

Ji Q (2004) Renormalization groups and critical phenomena. Nucl Phys Rev 21(2):174-176

Ji HG, Jiang H, Song ZY et al (2018) Analysis on the microstructure evolution and fracture morphology during the softening process of weakly cemented sandstone. J China Coal Soc 43(4):993-999

Li XC, Feng ZC, Guo JZ et al (2019) Study on influence laws of temperature and stress on sandstone permeability. Coal Sci Technol 47(4):96-100
Liu HT, Qin T (2019) Study on damage characteristics and acoustic emission Kaiser effect of sandstone under cyclic loading and unloading conditions. Coal Sci Technol 47(6):73-80

Liu S, Yang GS, Dong XH (2019) Experimental study on influence of wetting-drying cycles on mechanical characteristics and damage of red sandstone. Coal Sci Technol 47(4):101-106

Qin JM, Zhang HW(2010) Microscopic theoretical model of critical state for granular materials. Rock Soil Mech 31(12):3697-3703

Rothenburg L, Kruyt NP (2004) Critical state and evolution of coordination number in simulated granular materials. Int J Solids Struct 41:5763-5774

Song ZY (2017) The analysis and application of mesoscopic structure characteristic and deformation and failure mechanism of weakly cemented sandstone. University of Science and Technology Beijing, Beijing

Song ZY, Ning FB (2018) Progress on the association between mesostructured parameters and micromechanical behaviors of weakly cemented rocks. Metal Mine 12:1-9

Song ZY, Ji HG, Jiang $\mathrm{H}$ et al (2018a) Influence of wetting-drying cycles on acoustic emission characteristics and microstructure deterioration of weak cementation stratum. J China Coal Soc 43(S1):96-103

Song ZY, Ji HG, Liu ZQ et al (2018b) Morphology and failure mechanism of the shear fracture surface of weakly cemented sandstone with water saturation. J China Coal Soc 43(9):2444-2451

Wei ZH (2012) Research on structural problems of geo-materials. Beijing Jiaotong University

Weinberg S (1995) The quantum theory of fields, vol 2. Cambridge University Press, Cambridge, p 111

Wilson KG (1971a) Re-normalization group and critical phenomena. I. Re-normalization group and the Kadanoff scaling picture. Phys Rev B 4(9):3174-3183

Wilson KG (1971b) Re-normalization group and critical phenomena. II. Phase-space cell analysis of critical behavior. Phys Rev B 4(9):3184-3205

Zeng P, Liu YJ, Ji HG et al (2017) Coupling criteria and precursor identification characteristics of multi-band acoustic emis-sion of gritstone fracture under uniaxial compression. Chin J Geotech Eng 39(03):509-517

Zhang HW, Qin JM (2008) Microscopic critical state based on valence of granular materials. Rock Soil Mech 29(4):865-870

Zhou CY, Li BT, Zhang XH et al (2015) Study on percolation threshold of red-layer soft rock failure process based on the renormalization group method. J Eng Geol 23(5):995-970 